\title{
RIGOROUS SOLUTIONS FOR THE SPANWISE LIFT DISTRIBUTION OF A CERTAIN CLASS OF AIRFOILS*
}

\author{
BY \\ OTTO LAPORTE \\ University of Michigan
}

1. Introduction. The problem of the spanwise lift distribution of an airfoil in a uniform stream of air leads, as was shown by Prandtl, ${ }^{1}$ to the following integrodifferential equation for the circulation $\Gamma(y)$ :

$$
\frac{1}{4 \pi V} \int_{-b / 2}^{+b / 2} \frac{d y^{\prime}}{y-y^{\prime}} \frac{d \Gamma}{d y^{\prime}}+\frac{2 \Gamma}{m c(y) V}=\alpha .
$$

In this equation $y$ is the span coordinate, and $-\frac{1}{2} b \leqq y \leqq \frac{1}{2} b ; V$ is the velocity of the air at infinity, its direction being parallel to the $x$-axis; $c(y)$ is the chord function determining the shape or planform of the airfoil; $m$ is a numerical constant, which the theory of wings of infinite span fixes at $2 \pi$ but which seems to have an experimental value in the vicinity of $5.5 ; \alpha$ is the geometric angle of attack, which for flat wings is a constant but for twisted wings is a given function of $y$. For a derivation, the reader is referred to the papers mentioned in footnote 1 ; we only wish to note here that the downwash velocity $w$ is essentially the first term of (1) and has the form

$$
w(y)=\frac{1}{4 \pi} \frac{2}{b} \int_{-1}^{+1} \frac{d \eta^{\prime}}{\eta-\eta^{\prime}} \frac{d \Gamma}{d \eta^{\prime}},
$$

where $\eta=2 y / b$ is a dimensionless span coordinate. Because of the singular integrand, the Cauchy principal value must be taken both in (1) and (2). Of fundamental importance, furthermore, is the elliptic wing of chord distribution

$$
c(y)=c_{0} \sqrt{1-\eta^{2}}
$$

for which $\Gamma(y)$ is also of elliptic shape while $w(y)$ is constant.

In the extensive literature dealing with solutions of (1) for a given planform $c(y)$, the following procedure is used almost always: the substitution $\eta=\sin \theta$ is introduced, and the chord function $c(y)$ is developed in a Fourier series. It is assumed that $\Gamma$ is also of this form, whence equation (1) is satisfied at a discrete number of $y$ values. This leads to a system of linear equations for the Fourier coefficients, the solution of which is usually extremely laborious.

In contrast to this method, the point of view adopted by Trefftz ${ }^{2}$ seems to me more powerful. This author considers the potential flow in the complex $y+i z$ plane

* Received Feb. 28, 1944.

1 L. Prandtl, Göttinger Nachrichten, 1918, 451, and 1919, 107. Also N.A.C.A. Report 116, 1921. See also the presentation in Mises and Friedrichs' Fluid dynamics, Brown University mimeographed lecture notes, 1941, p. 108 and foll.

2 E. Trefftz, Zeitschr. f. Angew. Math. und Mechanik, 1, 206 (1921). 
at a large distance behind the wing. The wake, stretching along the $y$-axis from $-b / 2$ to $+b / 2$ becomes a line of discontinuity along which the velocity potential suffers a jump determined by the circulation. The integro-differential equation.(1) reveals itself as a boundary condition which the velocity potential has to obey along the line $-b / 2 \leqq y \leqq b / 2$. "With the aid of conformal transformation the field is brought into relation with the field outside of a circle of unit radius; then the potential is approximated by a trigonometric expression and an approximate fulfillment of the boundary condition is sought. ${ }^{3}$

In the present paper the point of view which was used by Trefftz is extended, but instead of "seeking an approximate fulfillment of the boundary condition" for an arbitrary chord function $c(y)$, a simultaneous choice of the function and of a conformal mapping function transforms the problem into a boundary value problem of classical type, which can be solved rigorously and in every detail. The resulting formulae lend themselves readily to numerical computation.

The family of planforms to which one is led in this fashion is represented by the equation

$$
c(y)=c_{0}\left[\left(1-\eta^{2}\right)\left(1-\kappa^{2} \eta^{2}\right)\right]^{1 / 2} .
$$

For

$$
0 \leqq \kappa \leqq 1,
$$

this results in airfoils of taper greater than in the elliptic case (3), while for

$$
0 \leqq \kappa \leqq i,
$$

airfoils blunter than the elliptic ones are obtained. Fig. 1 shows the entire family for a fixed span and aspect ratio. ${ }^{4}$

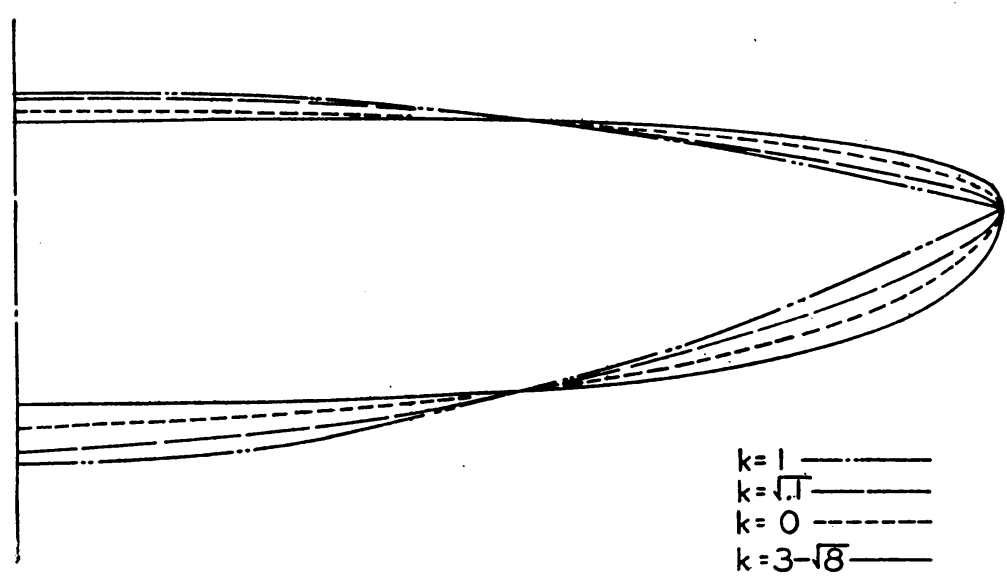

FIG. 1: $A=8$.

To be sure, for a given planform elaborate approximate methods, such as the Fourier series method described above, will probably always have to be used. How-

'v. Kármán and Burgers, in vol. II of Durand's Aerodynamic theory, Springer, Berlin 1935, p. 171.

4 The aspect ratio $A$ is defined as $b^{2} / S$, where $S$ is the area of the wing. For the calculation of $S$ as a function of $\kappa$, see (22). 
ever, we shall now show that at least for one planform family, which depends on two parameters, the problem can be completely solved. It is hoped that the method used here will be generalizable so as to furnish perhaps other chord functions in the future. At any rate, for a planform which is close to one of family (4) a method of successive approximations can be readily set up.

It seems that a comparison with the methods used in the theory of wings of infinite span is not superfluous. Here rigorous solutions are available for certain families of simple profiles, the simplest one of which is that of Joukowski. Although the designer will probably not be satisfied with such a simplification and will turn to more elaborate methods, there is an advantage in having easily derived formulae in closed form-both as far as quick estimates and classroom presentation are concerned. In lectures it is standard procedure to present the flow around the Joukowski and perhaps also around the Kármán-Trefftz profiles before turning to more general methods; on the other hand, when dealing with wings of finite span, the derivation of Prandtl's equation (1) is usually followed only by a detailed discussion of the elliptic case and then by a mere description of the Fourier series methods. The formulae given below are intended to fill this gap.

2. Prandtl's equation as a boundary condition in the complex plane $u=y+i z$. A brief recapitulation of Trefftz's work ${ }^{2}$ is necessary following the presentation of Mises and Friedrichs. ${ }^{1}$ In the $y+i z$ plane there exists a flow derivable from a potential $\phi(y, z)$. This potential is everywhere continuous except on the projection of the airfoil

$$
z=0, \quad-b / 2 \leqq y \leqq+b / 2,
$$

where, because of the existence of a circulation, $\phi$ is discontinuous. Assuming that the values of $\phi$ at opposite points of the slit (5) are equal and opposite, we have

$$
\Gamma(y)=4 \phi(y, 0) \text {. }
$$

The downwash velocity $w$ becomes

$$
w(y)=\left(\frac{\partial \phi}{\partial z}\right)_{0} .
$$

The problem is therefore to find a solution of the Laplace equation

$$
\Delta \phi=0
$$

which on the upper side of the slit (5) satisfies the boundary condition

$$
\left(\frac{\partial \phi}{\partial z}\right)_{0}-\frac{8 \phi(y, 0)}{m c(y)}=-V \alpha(y) .
$$

This condition results from substituting (2), (6) and (7) into (1). The corresponding condition on the lower side of the slit differs from (9) in that the sign of the second term is opposite.

Condition (9) characterizes the present problem as a boundary value problem of the third kind. As mentioned earlier, Trefftz now maps the region outside slit (5) into the interior of the unit circle by means of

$$
u=\frac{b}{4}\left(t+\frac{1}{t}\right)
$$


Through the introduction of polar coordinates $t=r(\cos \theta+i \sin \theta)$ he is then led to approximate solutions having the form of a Fourier series in $\theta$.

It is evident that, because of the occurrence in (9) of the function $c(y)$ in the coefficient of $\phi(y, 0)$, exact solutions of the problem will in general be difficult to obtain. But let us also examine the effect of a conformal mapping upon (8) and (9). While the former is invariant under arbitrary conformal transformations the latter is not, because of the occurrence of the first derivative. One may say that each conformal transformation causes a new variable coefficient to appear both in the $\phi$ term on the left and on the right side of (9).

We propose to let these two effects counteract one another and to choose both appropriate conformal transformations and appropriate chord functions $c(y)$ so as to arrive at a boundary condition with constant coefficients which lends itself to treatment by classical methods. An example will best serve to illustrate this procedure.

Instead of introducing real polar coordinates within the circle into which the slit (5) is mapped by (10), we shall map the interior of the circle onto the strip bounded by the points $0,+\infty,+\infty+2 \pi i, 2 \pi i$ of a $\lambda$ plane by means of

$$
t=e^{\lambda}, \quad u=\frac{b}{2} \operatorname{ch} \lambda,
$$

where $\lambda=\mu+i \nu$. The potential must be a solution of the Laplace equation in $\lambda$ and $\mu$, and must satisfy the following boundary condition along the imaginary axis on to which the circle $|t|=1$ is mapped:

$$
\left(\frac{\partial \phi}{\partial \mu}\right)_{0}-\frac{4 b \sin \nu}{m c\left(\frac{1}{2} b \cos \nu\right)} \phi(0, \nu)=-\frac{1}{2} b V \alpha \sin \nu .
$$

In order that the coefficient of $\phi$ be a constant we must choose $c\left(\frac{1}{2} b \cos \nu\right)$ proportional to $\sin \nu$, whence, by virtue of (11), we have the elliptic chord distribution (3).

3. A transformation mapping the interior of the circle $|t|=1$ into that of a rectangle. We now replace the function (11) by others which map the interior of the unit circle into various other regions. Each time, in order to have a boundary condition with constant coefficients, an appropriate chord function must be chosen. When mapping the unit circle of the $t$ plane into a rectangle in the usual Schwartzian way such that four arbitrarily chosen points on the periphery correspond to the corners, a chord function results which possesses singularities at those points of the span which are the images of the corners. It is, however, possible to map a rectangle onto the unit circle, such that two opposite sides of the rectangle become two opposite semicircular arcs while each of the two other sides of the rectangle map onto a slit protruding radially part way into the circle. ${ }^{5}$ This is illustrated in Fig. 2. The mapping function which accomplishes this is

$$
t=\sqrt{k} \text { sn } \frac{2 K}{\pi} Z,
$$

where the circle is in the $t$-plane $(t=r+i s)$, the rectangle is in the $Z$-plane $(Z=X+i Y)$,

${ }^{5} \mathrm{G}$. Holzmüller, Einführung in die Theorie der isogonalen Verwandtschaften, 1882, p. 256 and foll. See also Cayley, Collected papers, vol. 13, papers No. 891, 920, and 921. 
$k$ is the modulus of the elliptic function and is between 0 and $1, K$ is the complete elliptic integral. We now separate real and imaginary parts by means of the addition theorem of the sn function and put

$$
Y= \pm \frac{\pi}{4} \frac{K^{\prime}}{K}
$$

In view of the fact that $\operatorname{sn}\left(i K^{\prime} / 2\right)=i k^{-1 / 2}$, we obtain readily

$$
r=(1+k) \frac{\operatorname{sn} 2 K X / \pi}{1+k \operatorname{sn}^{2} 2 K X / \pi} \quad s= \pm \frac{\operatorname{cn} 2 K X / \pi \operatorname{dn} 2 K X / \pi}{1+k \operatorname{sn}^{2} 2 K X / \pi} .
$$

Therefore

$$
r^{2}+s^{2}=1 .
$$

Thus the upper straight line (14) maps into the upper semicircle, and lower one into
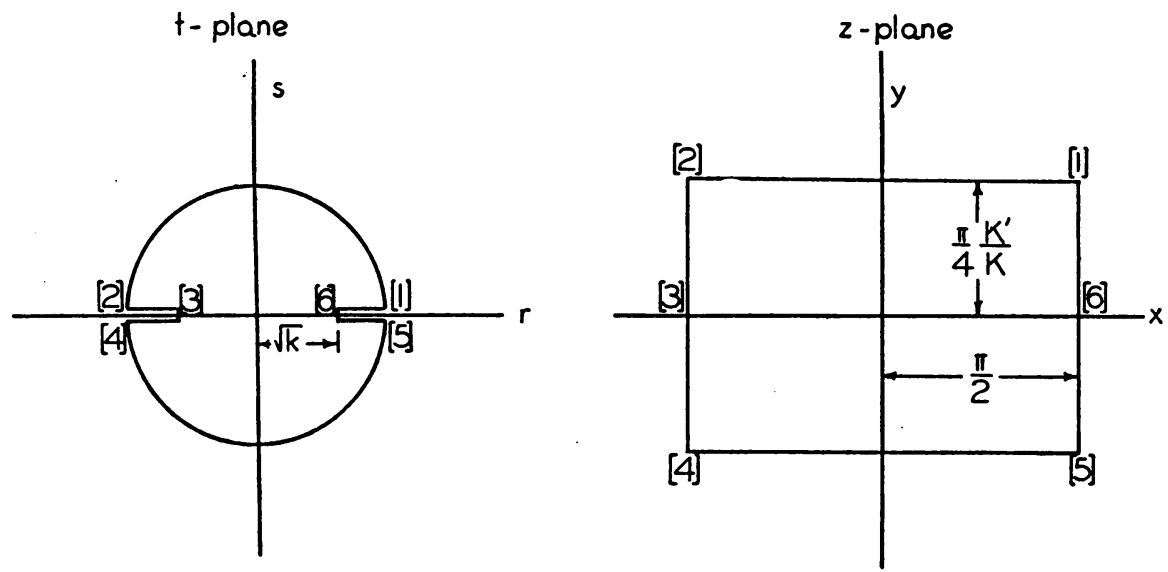

FIG. 2.

the lower. If in (15) we set $X= \pm \frac{1}{2} \pi$, we obtain $s=0$. The four corners of the rectangle are hereby fixed. To see what corresponds to the vertical sides, we set $Z= \pm \pi / 2+i Y$ in (13), to obtain

$$
r= \pm \sqrt{k} \text { nd }\left(\frac{2 K}{\pi} Y, k^{\prime}\right)
$$

where $k^{\prime 2}=1-k^{2}$. The slits protruding into the circle along the real axis therefore terminate at $r= \pm k^{1 / 2}$.

4. The boundary condition in the $Z$-plane. For the upper side of the rectangle, this condition becomes, by use of (9), (10) and (13),

$$
\left(\frac{\partial \phi}{\partial Y}\right)_{\pi K^{\prime} / 4 K}+\frac{8}{m c}\left(\frac{d u}{d Z}\right)_{\pi K^{\prime} / 4 K} \phi\left(X, \frac{\pi}{4} \frac{K^{\prime}}{K}\right)=V \alpha\left(\frac{d u}{d Z}\right)_{\pi K^{\prime} / 4 K}
$$

where

$$
\frac{d u}{d Z}=\frac{b}{2 \pi} \sqrt{k} K\left(1-k^{-1} \mathrm{~ns}^{2} \frac{2 K}{\pi} Z\right) \mathrm{cn} \frac{2 K}{\pi} Z \operatorname{dn} \frac{2 K}{\pi} Z
$$

and $Z$ must be put equal to $X+i \pi K^{\prime} / 4 K$. The chord $c(y)$, now regarded as a func- 
tion of $X$, must be chosen proportional to (19). Therefore it becomes necessary to express (19) as a function of $y$ in the original $u$ plane. We have at first from (13),

$$
\frac{d u}{d Z}=\frac{b \sqrt{k} K}{2 \pi}\left(1-\frac{1}{t^{2}}\right)\left[\left(1-\frac{t^{2}}{k}\right)\left(1-k t^{2}\right)\right]^{1 / 2} .
$$

where $|t|=1$. Using for the moment the angle $\nu$ as in (12), this may be written in the form

$$
\frac{d u}{d Z}=\frac{i b \sqrt{k} K}{\pi} \sin v\left[2 \cos 2 v-\left(k+\frac{1}{k}\right)\right]^{1 / 2}
$$

which, since $\cos \nu=\eta$, becomes finally

$$
\frac{d u}{d Z}=\frac{1}{\pi} b K(1+k)\left[\left(1-\eta^{2}\right)\left(1-\kappa^{2} \eta^{2}\right)\right]^{1 / 2} .
$$

The abbreviation

$$
\frac{1}{\kappa}=\frac{1}{2}\left(\frac{1}{\sqrt{k}}+\sqrt{k}\right)
$$

was used, where $\kappa$ is between 0 and 1. We see thus that we are led to the planforms (4), (4') which represent wings of taper greater than the elliptic wing.

Before (4) and (20) are substituted into (18), it is convenient to calculate the area $S$ and the aspect ratio $A=b^{2} / S$. It stands to reason that the area

$$
S=\int_{-b / 2}^{b / 2} c_{0}\left[\left(1-\eta^{2}\right)\left(1-\kappa^{2} \eta^{2}\right)\right]^{1 / 2} d y
$$

is expressible in terms of the complete elliptic integrals $E$ and $K$ of $\kappa$, but in view of the fact that (21) is the well known Landen transformation, $S$ can also be reduced to the complete elliptic integrals of the modulus $k$. The result is

where

$$
S=b c_{0} G(k),
$$

$$
G(k)=\frac{1}{6 k}\left[\frac{1+6 k+k^{2}}{1+k} E(k)-(1-k)(1-3 k) K(k)\right] .
$$

$G(k)$ is a purely numerical constant depending on taper. For the aspect ratio we have

and for the average chord $\bar{c}$,

$$
\mathcal{A}=\frac{b}{G(k) c_{0}}
$$

$$
\bar{c}=G(k) c_{0} .
$$

Substitution of (4), (20) and (23) into (18) gives the final form of the boundary condition,

$$
\begin{aligned}
& \left(\frac{\partial \phi}{\partial Y}\right)_{K^{\prime} / 4 K}+\frac{8}{\pi} \frac{\mathcal{A}}{m}(1+k) G K \phi\left(X, \frac{\pi}{4} \frac{K^{\prime}}{K}\right) \\
& =\frac{V b}{2 \pi} \sqrt{k} K\left[\alpha\left(1-k^{-1} \mathrm{~ns}^{2} \frac{2 K}{\pi} Z\right) \operatorname{cn} \frac{2 K}{\pi} Z \operatorname{dn} \frac{2 K}{\pi} Z\right]_{Y=\pi K^{\prime} / 4 K} .
\end{aligned}
$$


Variable coefficients now only occur on the right. It should be noted that for $Z=X+i \pi K^{\prime} / 4 K$ the right-hand side represents a real function of $X$.

5. Solution of the boundary value problem for tapered wings. This solution has to satisfy the following requirements: tion.

(1) Inside the rectangle $|X| \leqq \pi / 2,|Y| \leqq \pi K^{\prime} / 4 K$, it must satisf y Laplace's equa-

(2) On the top side $Y=\pi K^{\prime} / 4 K$ it must satisfy (24).

(3) On the lower side it must satisfy a similar condition differing from (24) only in the sign of the first term.

We shall seek a solution of the form

$$
\phi(X, Y)=\sum_{n} \operatorname{sh} n Y\left(A_{n} \cos n X+B_{n} \sin n X\right) .
$$

In this series each term is a solution of the Laplace equation. The sh function of $Y$ is alone present because of condition (3), or in other words, because we want $\phi$ to have equal and opposite values at opposite points on slit (5) in the $y+i z$ plane. To determine $A_{n}$ and $B_{n}$ the right-hand side of (24), considered as a function of $X$, has to be expanded in a Fourier series.

At this moment it should be remembered that for twisted airfoils $\alpha$ is a function of $y$ or $\eta$. It will be an even function as long as the ailerons are in their normal position. Otherwise, $\alpha$ may have an odd component or may even be discontinuous. At the present time only the case of constant $\alpha$ will be treated."

To develop the right-hand side of (24) into a Fourier series we begin with the familiar series ${ }^{7}$ for $\mathbf{s n}$ :

$$
\operatorname{sn} \frac{2 K}{\pi} Z=\sum_{n=0}^{\infty} a_{n} \sin (2 n+1) Z, \quad a_{n}=\frac{\pi}{K k} \operatorname{cosech} \pi\left(n+\frac{1}{2}\right) \frac{K^{\prime}}{K} .
$$

This series converges uniformly as long as $|I(z)|<\pi K^{\prime} / 2 K$, i.e., within a horizontal strip whose median line is the real axis and whose width is $\pi K^{\prime} / K$. But since this is just twice the vertical dimension of the rectangle of the boundary value problem (see Fig. 2), series (26) will certainly converge absolutely anywhere that it is needed at present. We differentiate and put $Z=i \pi K^{\prime} / 4 K+X$, to obtain

$$
\operatorname{cn} \frac{2 K}{\pi} Z \operatorname{dn} \frac{2 K}{\pi} Z=\frac{\pi}{2 K} \sum_{n=0}^{\infty}(2 n+1) a_{n} \cos (2 n+1)\left(i \frac{\pi}{4} \frac{K^{\prime}}{K}+X\right) .
$$

To get the other part of the right-hand side of (24), we put $Z=i \pi K^{\prime} / 2 K+Z^{\prime}$, whence

$$
\mathrm{ns} \frac{2 K}{\pi} Z=k \mathrm{sn} \frac{2 K}{\pi} Z^{\prime}
$$

\footnotetext{
- More general cases of twisted airfoils are being computed at present and will be reported on elsewhere.

'See, for instance. Whittaker and Watson, Modern analysis, 4th ed., Cambridge University Press, p. 510.
} 
The application of (26) with $Z^{\prime}$ as the variable gives

$$
k^{-1} \mathrm{~ns} \frac{2 K}{\pi} Z=\sum_{n=0}^{\infty} a_{n} \sin (2 n+1) Z^{\prime} .
$$

After differentiating with respect to $Z$ or $Z^{\prime}$, we put $Z^{\prime}=-i \pi K^{\prime} / 4 K+X$ (which is the same as putting $\left.Z=+i \pi K^{\prime} / 4 K+X\right)$, to obtain

$$
-k^{-1} \mathrm{cs} \frac{2 K}{\pi} Z \mathrm{ds} \frac{2 K}{\pi} Z=\frac{\pi}{2 K} \sum_{n=0}^{\infty}(2 n+1) a_{n} \cos (2 n+1)\left(-i \frac{\pi}{4} \frac{K^{\prime}}{K}+X\right) .
$$

The sum of (27) and (28) furnishes the desired series which, because of the second formula of (26), may be written in the form

$$
\left(1-k^{-1} \mathrm{~ns}^{2} \frac{2 K}{\pi} Z\right) \mathrm{cn} \frac{2 K}{\pi} Z \operatorname{dn} \frac{2 K}{\pi}=\frac{\pi^{2}}{2 K^{2} k} \sum_{n=0}^{\infty}(2 n+1) \frac{\cos (2 n+1) X}{\operatorname{sh}\left(n+\frac{1}{2}\right) \pi K^{\prime} / 2 K},
$$

where it is to be understood that $Z=i \pi K^{\prime} / 4 K+X$. This series converges uniformly for all real values of $X$.

The final solution of the problem can now be written down. Introducing (29) and (25) into (24) one obtains for the velocity potential,

where

$$
\phi(X, Y)=\frac{\pi}{4} \frac{V \alpha b}{K \sqrt{k}} \sum_{n=0}^{\infty} \frac{\operatorname{sh}(2 n+1) Y \cos (2 n+1) X}{D_{n} \operatorname{sh}(2 n+1) \pi K^{\prime} / 4 K},
$$

$$
D_{n}=\operatorname{ch}(2 n+1) \frac{\pi}{4} \frac{K^{\prime}}{K}+\frac{8}{\pi} \frac{A}{m} \frac{(1+k) G K}{2 n+1} \operatorname{sh}(2 n+1) \frac{\pi}{4} \frac{K^{\prime}}{K} .
$$

This series converges uniformly as long as $|Y|<\pi K^{\prime} / 2 K$, which is more than we need for numerical calculations, since the rectangle only extends to $Y= \pm \pi K^{\prime} / 4 K$.

Of greater practical importance than $\phi$ is the sectional lift or the sectional lift coefficient $c_{l}$ which may be defined as follows: let $l$ be the lift per unit span,

$$
l=\frac{1}{2} \rho V^{2} \bar{c} c_{l},
$$

where $\bar{c}$ is the average chord $\left(23_{1}\right)$. Since from (6) $l$ is also $4 \rho V \phi\left(X, \pi K^{\prime} / 4 K\right)$, the following expression results:

$$
c_{l}=2 \pi \alpha \frac{A}{K \sqrt{ } \bar{k}} \sum_{n=0}^{\infty} \frac{1}{D_{n}} \cos (2 n+1) X .
$$

For this rapidly converging series, numerical tests have shown that three or four terms suffice to give a result correct to one part in ten thousand. To formula (33) should be added the relation between $X$ and the original span coordinate $y$. From (15) and (10) it is found to be

$$
y=\frac{b}{2}(1+k) \frac{\operatorname{sn} 2 K X / \pi}{1+k \operatorname{sn}^{2} 2 K X / \pi} .
$$

Formulae (33) and (34) give the spanwise lift distribution as a function of $y$. 
6. Results for the blunt wing. The method of the previous section may readily be used to get similar results for wings blunter than elliptic ones. The underlying idea is the following: in section 2 we mapped the $t$ circle onto a square such that the semicircle in the upper half plane became the upper side of a rectangle in the $Z$ plane, and correspondingly for the lower semicircle. Now, the interior of the unit circle in the $t$ plane will be mapped so that the right and left semicircles will correspond to the right and left sides of the rectangle, while two slits from $\pm i$ to $\pm i \sqrt{k}$ will correspond to the upper and lower sides. In short, the role on the real and imaginary axes in both planes will be reversed. Results will merely be given and formulas will receive the same numbers as the corresponding formula of the "tapered" case, except that primes will be added.

The mapping just described is performed by

$$
t=i^{-1} \sqrt{k} \text { sn } \frac{2 K}{\pi} i Z .
$$

Upon putting $X= \pm \pi K^{\prime} / 4 K$ the point in the $t$-plane moves on a circle $r^{2}+s^{2}=1$,

t-PLANE

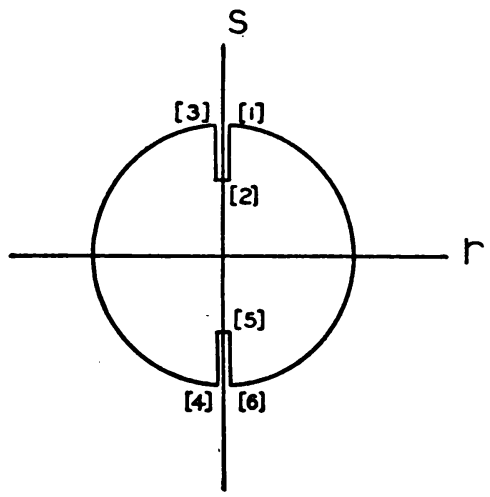

Z-PLANE

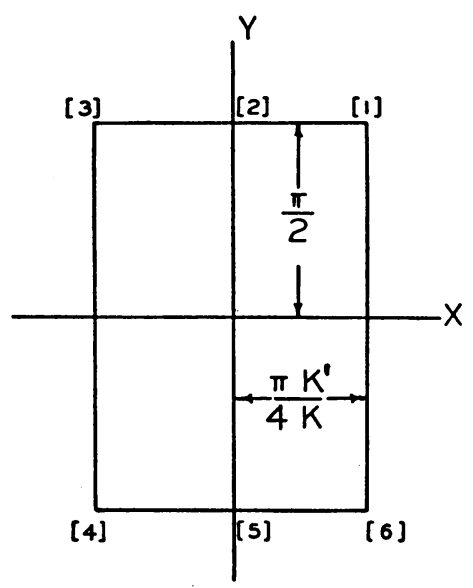

Fig. 3.

while $Y= \pm \pi / 2$ gives the slits along the imaginary axis of $t$. This is shown in Fig. 3 . The interior of the unit circle of the $t$ plane is then mapped by means of (10) onto the exterior of the slit (5) in the $u$ plane.

The boundary condition, now along the two vertical sides, becomes

with

$$
\left(\frac{\partial \phi}{\partial X}\right)_{\pi K^{\prime} / 4 K}+\frac{8}{i m c}\left(\frac{d u}{d Z}\right)_{\pi K^{\prime} / 4 K} \phi\left(\frac{\pi}{4} \frac{K^{\prime}}{K}, Y\right)=i^{-1} V \alpha\left(\frac{d u}{d Z}\right)_{\pi K^{\prime} / 4 K},
$$

$$
\left(\frac{d u}{d Z}\right)_{\pi K^{\prime} / 4 K}=\frac{b}{2 \pi} \sqrt{k} K\left(1+k^{-1} \mathrm{~ns}^{2} \frac{2 K}{\pi} i Z\right) \mathrm{cn} \frac{2 K}{\pi} i Z \mathrm{dn} \frac{2 K}{\pi} i Z,
$$

which is imaginary for $Z=\pi K^{\prime} / 4 K+i Y$. The determination of the planform results from expressing this in terms of $\eta$ : 


$$
i^{-1}\left(\frac{d u}{d Z}\right)_{\pi K^{\prime} / 4 K}=\frac{1}{\pi} b K(1-k) \sqrt{\left(1-\eta^{2}\right)\left(1+\kappa^{\prime 2} \eta^{2}\right)}
$$

where

$$
\frac{1}{\kappa^{\prime}}=\frac{1}{2}\left(\frac{1}{\sqrt{k}}-\sqrt{k}\right)
$$

The different signs should be noted. The two functions $\kappa$ of $\kappa^{\prime}$ of $k$ are plotted in Fig. 4. In this way a planform blunter than the elliptic one results, namely $(4),\left(4^{\prime \prime}\right)$. When $\kappa^{\prime}$ becomes greater than one, the chord function has a maximum between $\eta=0$ and 1 . The bluntest wing shape without such a maximum is attained for $\kappa^{\prime}=1$ or

$$
k_{C R}=3-\sqrt{8},
$$

in which case the lemniscate functions result. Thus the formulae of this section, while being valid for any $k$ between 0 and 1 have aerodynamical importance only for $k$ between 0 and $k_{C R}{ }^{8}$

The area of the wing becomes

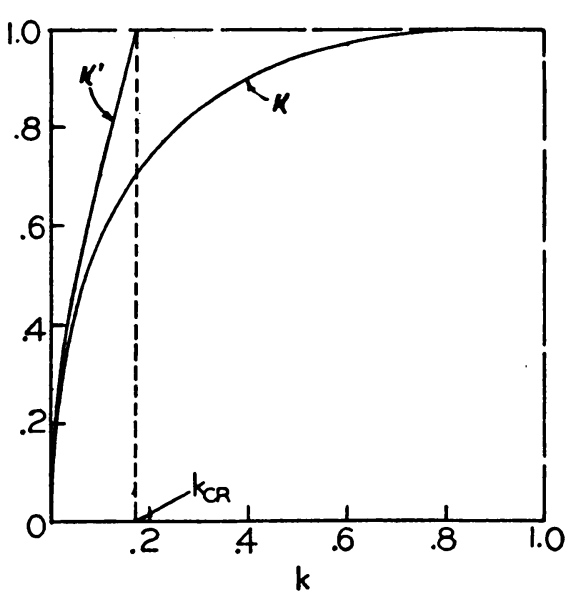

FIG. 4.

$$
S=b c_{0} G(-k),
$$

where $G(-k)$ is the function in equation $\left(22_{1}\right)$ for negative $k .{ }^{9}$ In the case of (34), which interests us especially, the elliptic integral becomes reducible to Gamma functions:

$$
G\left(-k_{C R}\right)=\frac{\Gamma(3 / 2) \Gamma(5 / 4)}{\Gamma(7 / 4)}=.87402 .
$$

Formulae for $\mathcal{A}$ and the mean chord $\bar{c}$ are taken over with $G(-k)$.

For a constant angle of attack, the only case treated below, the function in (19') will be developed in a Fourier series on the vertical sides $X= \pm \pi K^{\prime} / 4 K$ of the rectangle. This is readily accomplished, the difference from (29) being that only terms in $\sin (2 n+1) Y$ occur. The final result for the velocity potential is

$$
\phi(X, Y)=\frac{\pi}{4} \frac{V \alpha b}{K \sqrt{k}} \sum_{n=0}^{\infty} \frac{\operatorname{ch}(2 n+1) X \sin (2 n+1) Y}{\Delta_{n} \operatorname{ch}(2 n+1) \pi K^{\prime} / 4 K},
$$

where

$$
\Delta_{n}=\operatorname{sh}(2 n+1) \frac{\pi}{4} \frac{K^{\prime}}{K}+\frac{8}{\pi} \frac{\mathcal{A}}{m} \frac{(1-k) G(-k) K}{2 n+1} \operatorname{ch}(2 n+1) \frac{\pi}{4} \frac{K^{\prime}}{K} .
$$
note 5).

8 The mapping for $k=3-\sqrt{8}$ is studied and illustrated by figures in Holzmüller's book (see foot-

$\bullet$ However, $E$ and $K$ depend only on $k^{2}$. 
Convergence properties are the same as before. The sectional lift coefficient (32) is given by

$$
c_{l}=2 \pi \alpha \frac{A}{K \sqrt{ } \bar{k}} \sum_{n=0}^{\infty} \frac{1}{\Delta_{n}} \sin (2 n+1) Y,
$$

while the relation between the variable $Y$ and span coordinate $y$ is now

$$
y=\frac{b}{2} \frac{\operatorname{cn} 2 K Y \operatorname{dn} 2 K Y / \pi}{1+k \operatorname{sn}^{2} 2 K Y / \pi} .
$$

7. The elliptic wing as a limiting case. From (20) or (21) it is evident that the chord function represents an ellipse when $k$ goes to zero. For decreasing $k$ the elliptic integrals $K$ and $G$ approach $\pi / 2$ and $\pi / 4$ respectively, while

$$
\lim _{k \rightarrow 0}\left(K^{\prime}-\log \frac{4}{k}\right)=0 .
$$

The quotient $K^{\prime} / K$ becomes large and the convergence of the series (33) improves. Keeping only the first term we see that

Thus

$$
\lim _{k \rightarrow 0}\left\{\left(\begin{array}{l}
\mathrm{ch} \\
\mathrm{sh}
\end{array}\right) \frac{\pi}{4} \frac{K^{\prime}}{K}-k^{-1 / 2}\right\}=0
$$

$$
c_{l}=\frac{4}{\pi} \alpha m \frac{\cos X}{1+\pi \mathcal{A} / m}
$$

which is in complete agreement with Durand II, p. 169, since now $\cos X=\left(1-\eta^{2}\right)^{1 / 2}$.

8. The limiting case of extreme taper, $k=1$. This case is of more interest than the preceding one, inasmuch as it leads to new and simple formulae in closed form. It follows from (20) that the planform is now given by

$$
c=c_{0}\left(1-\eta^{2}\right) \text {, }
$$

which represents a parabolic arc or two such arcs joined along the $y$-axis (see Fig. 1). Although the lift distribution can be obtained from (33) by letting $k$ tend towards 1 , we prefer to derive it afresh.

The transformation

$$
t=\tanh Z
$$

maps the interior of the unit circle in the $t$ plane onto a strip parallel to the $X$-axis and bounded by $Y= \pm \pi / 4$. The points $t= \pm 1$, where the $t$ circle crosses the real axis move, respectively, towards $+\infty$ and $-\infty$. Transformations (10) and (39) together map the outside of slit (5) in the $u$ plane on the above mentioned strip, in such a way that the upper and lower sides of the slit become, respectively, the upper and lower sides of the strip, while the tips $(y= \pm b / 2)$ move to infinity.

The boundary condition on the upper side is the same as equation (18), except that the subscript on the various derivatives now is $Y=\pi / 4$. Since the transformation from the $u$ to the $Z$ plane is now

$$
u=\frac{1}{2} b \operatorname{coth} 2 Z,
$$

as is seen from (39) and (10), we have as the analogue of (19) the equation 


$$
\left(\frac{d u}{d Z}\right)_{Y=\pi / 4}=b \operatorname{sech}^{2} 2 X,
$$

which, when expressed as function of $\eta$, becomes $b\left(1-\eta^{2}\right)$. Hence the chord is chosen as in (38). We note the formulae for mean chord $\bar{c}$, area $S$, and aspect ratio $\mathbb{R}$ :

$$
\bar{c}=\frac{2}{3} c_{0} ; \quad S=\frac{2}{3} b c_{0} ; \quad \mathcal{A}=\frac{3}{2} \frac{b}{c_{0}} .
$$

The final form of the boundary condition on the upper side of the infinite strip is

$$
\left(\frac{\partial \phi}{\partial Y}\right)_{\pi / 4}+\frac{16}{3} \frac{\mathcal{A}}{m} \phi\left(X, \frac{\pi}{4}\right)=V \alpha b \operatorname{sech}^{2} 2 X .
$$

Due to the fact that the range of $X$ is from $-\infty$ to $+\infty$, the right-hand side of (43) must now be expanded in a Fourier integral. This can be done by standard methods, the result being

$$
\operatorname{sech}^{2} 2 X=\frac{1}{4} \int_{0}^{\infty} \frac{\zeta d \zeta \cos \zeta X}{\operatorname{sh} \pi \zeta / 4} .
$$

For the velocity potential $\phi(X, Y)$ we assume a solution of the form

$$
\phi(X, Y)=\int_{0}^{\infty} d \zeta \dot{A(\zeta)} \operatorname{sh} \zeta Y \cos \zeta X,
$$

where the integrand satisfies the Laplace equation. After introducing (45) and (44) into (43), $A(\zeta)$ is easily determined. The final form for $\phi(X, Y)$ is

$$
\phi(X, Y)=\frac{1}{4} V \alpha b \int_{0}^{\infty} \frac{\operatorname{sh} \zeta Y \cos \zeta X}{D(\zeta) \operatorname{sh} \pi \zeta / 4} d \zeta,
$$

where

$$
D(\zeta)=\operatorname{ch} \frac{\pi}{4} \zeta+\frac{16}{3} \frac{A}{m} \frac{\operatorname{sh} \pi \zeta / 4}{\zeta} .
$$

The sectional lift coefficient assumes the form

$$
c_{\imath}=2 \mathcal{A} A \int_{0}^{\infty} \frac{\cos \zeta X}{D(\zeta)} d \zeta .
$$

The integral in (46) converges for all $X$ and $Y$ within the strip under consideration. In order to obtain from (48) the lift distribution as a function of $y$, we note here that, due to (40),

$$
y=\frac{1}{2} b \tanh 2 X .
$$

The above integral may be brought into a rather different and interesting form which makes the numerical evaluation very much easier. Let us consider the integral

$$
\int_{-\infty}^{+\infty} \frac{\tau d \tau \cos \tau \xi}{\tau \operatorname{ch} \tau+a \operatorname{sh} \tau}
$$

which, except for a simpler notation, is the same as that in (48). On the imaginary $\tau$ 
axis, the integrand has infinitely many poles, which can be computed as the roots of the transcendental equation

$$
\sigma \cos \sigma+a \sin \sigma=0 \text {. }
$$

Denoting the $n$th root by $\sigma_{n}$, we find from this that

$$
\cos \sigma_{n}=(-1)^{n} a\left(a_{n}+\sigma_{n}^{2}\right)^{-1 / 2}, \quad \sin \sigma_{n}=(-1)^{n+1} \sigma_{n}\left(a^{2}+\sigma_{n}^{2}\right)^{-1 / 2} .
$$

For $\tau=0$ the integrand is regular. It now becomes convenient to decompose the cos in the numerator into two exponentials. Two integrals arise in this way, one containing $\exp i \tau \xi$, the other $\exp (-i \tau \xi)$. According to a standard argument, the integral with the positive exponential tends to zero when integrated along the circumference of a large semicircle in the upper half plane, as the radius increases; similarly for the other integral and a semicircle around the origin in the lower half plane. Thus the integral with the positive exponential, taken along the real axis from $-\infty$ to $+\infty$, is equal to the sum of the residues at the poles $\sigma_{n}$ which lie in the upper half plane, and the integral with the negative exponentials is equal to the sum of the residues at the poles of the lower half plane.

Applying these considerations to (48) we see that the quantity $a$, which determines the roots of the transcendental equation, has the value

$$
a=\frac{8}{3} \frac{\mathcal{A}}{m}
$$

while the sectional lift coefficient is given by

$$
c_{l}=8 \alpha \mathcal{A} \sum_{n=0}^{\infty}(-1)^{n+1} \frac{\sigma_{n}\left[a^{2}+\sigma_{n}\right]^{1 / 2}}{a(a+1)+\sigma_{n}^{2}} e^{-4 \sigma_{n} X / x} .
$$

This is a very rapidly converging series, which, although especially convenient near the wing tips, may be used to advantage for values of $\eta$ as small as .3 or .2. Very near the wing tips $X$ is approaching $\infty$. Hence, using (49) we may write approximately

$$
c_{l}=8 \alpha A \frac{\sigma_{1}\left[a^{2}+\sigma_{1}^{2}\right]^{1 / 2}}{a(a+1)+\sigma_{1}^{2}}\left(\frac{1-\eta}{2}\right)^{\sigma_{1} / \pi},
$$

which shows that $c_{l}$ has a vertical tangent at the tips since $\sigma_{1}$ is between $\pi / 2$ and $\pi$.

The only inconvenient region is that of small $X$, especially the point $X=0$. It seems that only graphic integration can be used to find $c_{l}$ at the center of the wing from formula (48).

9. Total lift and total induced drag. For these important quantities rapidly convergent expressions are readily derived. The total lift $L$ is obtained by integrating $\rho V \Gamma$ across the span,

$$
L=4 \rho V \int_{-b / 2}^{+b / 2} \phi(y, 0) d y
$$

and transforming to the $Z$ plane, to obtain

$$
L=4 \rho V \int_{-\pi / 2}^{+\pi / 2} \phi\left(X, \frac{\pi}{4} \frac{K^{\prime}}{K}\right)\left(\frac{d u}{d Z}\right)_{\pi K^{\prime} / 4 K} d X
$$


The two Fourier series for $\phi$ and $d u / d Z$ are then substituted from (30), (19) and (29). From the completeness relation it follows that the integral of the product of the two series is equal to the sum of the products of the coefficients. Instead of the formula for $L$, merely the final formula for the over-all lift coefficient $c_{L}$, defined by $L=\frac{1}{2} \rho V^{2} S c_{L}$, will be given: $:^{10}$

$$
c_{L}=\frac{\pi^{3}}{4} \frac{A \alpha}{K^{2} k} \sum_{n=0}^{\infty} \frac{2 n+1}{D_{n} \operatorname{sh}(2 n+1) \pi K^{\prime} / 4 K} .
$$

The abbreviation $D_{n}$ was defined in (31). In the limit of vanishing $k$ this reduces exactly to formula (2.15), p. 169 of Durand, Vol. II.

For wings blunter than elliptic ones the result is

$$
c_{L}=\frac{\pi^{3}}{4} \frac{\mathcal{A}}{K^{2} k} \sum_{n=0}^{\infty} \frac{2 n+1}{\Delta_{n} \operatorname{ch}(2 n+1) \pi K^{\prime} / 4 K},
$$

where $\Delta_{n}$ is the coefficient defined in $\left(31^{\prime}\right)$.

For the parabolic wing treated in section 7 , it is necessary to go through the operation just described for the Fourier integrals (44) and (46). The lift coefficient is now

$$
c_{L}=\frac{\pi}{2} A \alpha \int_{0}^{\infty} \frac{\zeta d \zeta}{D(\zeta) \operatorname{sh} \pi \zeta / 4} .
$$

For the induced drag $D_{i}$ similarly simple formulae may be obtained. Using (7) we have first in the original $u$-plane $(u=y+i z)$

$$
D_{i}=4 \rho \int_{-b / 2}^{b / 2} \phi(y, 0)\left(\frac{\partial \phi}{\partial Z}\right)_{0} d y .
$$

In the $Z$ plane of the tapered wing this may be written

$$
D_{i}=4 \rho \int_{-\pi / 2}^{\pi / 2} \phi\left(X, \frac{\pi}{4} \frac{K^{\prime}}{K}\right)\left(\frac{\partial \phi}{\partial Y}\right)_{\pi K^{\prime} / 4 K} d X .
$$

Now the completeness relation must be applied to the Fourier series (30) and its own derivative. The result is, expressed in terms of the drag coefficient,

$$
c_{D}=\frac{\pi^{3}}{4} \frac{\alpha^{2} \mathcal{A}}{K^{2} k} \sum_{n=0}^{\infty} \frac{2 n+1}{D_{n}^{2}} \operatorname{coth}(2 n+1) \frac{\pi}{4} \frac{K^{\prime}}{K},
$$

while for the blunt wing we have

$$
c_{D}=\frac{\pi^{3}}{4} \frac{\alpha^{2} \mathcal{A}}{K^{2} k} \sum_{n=0}^{\infty} \frac{2 n+1}{\Delta_{n}^{2}} \tanh (2 n+1) \frac{\pi}{4} \frac{K^{\prime}}{K},
$$

${ }^{10}$ Formulae (53) and (54) may be derived in a different way without appealing to the completeness relation. The velocity potential $\phi(y, z)$ is only the real part of a complex stream function $F(u)$ in terms of which the total lift may be written in the form

$$
L=2 \rho V \operatorname{Re} \oint F(u) d u \text {. }
$$

When transformed into the $Z$-plane, this becomes a contour integral around the rectangle of Fig. 2. This integral can be carried out simply by applying Cauchy's theorem. The same can be done for the rolling moment. This method recalls the Blasius theorem in the theory of infinite span. 
and for the parabolic wing

$$
c_{D}=\frac{\pi}{2} \alpha^{2} \mathcal{C} \int_{0}^{\infty} \frac{\zeta d \zeta}{D^{2}(\zeta)} \operatorname{coth} \frac{\pi \zeta}{4} .
$$

Formulae (53) to (56) give the dependence of lift and induced drag upon aspect ratio $\mathcal{A}$ and taper $k$. However, it is not necessary to compute more than $c_{L}$, for there exists the following simple relation between $c_{L}$ and $c_{D}$ :

$$
c_{D}=\alpha \mathcal{A} \frac{\partial c_{L}}{\partial \mathcal{A} A} .
$$

Thus after having plotted a set of curves showing the dependence of $c_{L}$ upon the aspect ratio, one may obtain $c_{D}$ by graphic differentiation.

Further over-all quantities of practical interest are the rolling moment

$$
4 \rho V \int_{-b / 2}^{b / 2} \phi(y, 0) y d y
$$

and the yawing moment

$$
4 \rho \int_{-b / 2}^{b / 2} \phi(y, 0)\left(\frac{\partial \phi}{\partial Z}\right)_{0} y d y,
$$

but since in this paper we have confined ourselves through out to constant angles of attack, they will vanish. However, when $\alpha(y)$ is assumed to have an odd component the methods described here still work and the above moments yield formulae of type (53) to (56).

10. Numerical results. All formulae given in the preceding sections for the sectional lift coefficients are readily evaluated, due to the fact that the series converge rapidly. An exception is $c_{l}$ for the parabolic wing shape for small or zero span coordinate, in which case it is necessary to resort to graphic integration. Following v. Kármán and Burgers, ${ }^{3}$ we plot $c_{l} / m \alpha$ as a function of the span coordinate $\eta$ rather than $c_{l}$ itself. In this way it is necessary to vary only the two parameters $\mathrm{A} / \mathrm{m}$ and the taper constant $k$.

In Tables 1 and 2 values of $c_{l} / m \alpha$ are given which are computed from formulae (33) and (34) for $k=\sqrt{ } .1$ and $k=\sqrt{ } .2$. In the first column are the values of $2 K X / \pi$ which quantity serves as a convenient parameter, in the second $X$, in the third $\eta$, computed by means of the tables by Milne-Thomson ${ }^{11}$. In the last three columns the values of $c_{l} / m \alpha$ are to be found for $\mathcal{A} / m$ equal to $1.0,1.5$ and 2.0 , corresponding to aspect ratios of about 5.5, 9.25 and 11 .

Among wings blunter than elliptic only the one with $k_{\mathrm{CR}}$ of (35) has been computed using formulae $\left(33^{\prime}\right)$ and $\left(34^{\prime}\right)$. However, since elliptic functions with $k$ values as small as $k_{\mathrm{CR}}$ were not tabulated by Milne-Thomson, equation (34') was expanded in powers of that small constant. Values of $c_{l} / m \alpha$ are given in Table 3.

Finally in Table 4 the same data are given for the parabolic wing of section 7 . In column 1 are the values of the parameter $X$, in column 2 the $\eta$ values obtained from it with (49), and in the next three columns the values of $c_{l} / m \alpha$. For $X=0, .1, .2$ they were obtained by graphical integration from (48), and for all greater values of $X$ from

' L. M. Milne-Thomson, Die elliptischen Funktionen von Jacobi, Springer, Berlin, 1931. 
(52). The roots of the transcendental equation (51) do not seem to have been calculated before, at least not for the values $\left(51^{\prime \prime}\right)$ of the constant $a$. They were determined to five significant figures.

These tables are supplemented by the following figures: Fig. 5 makes possible a

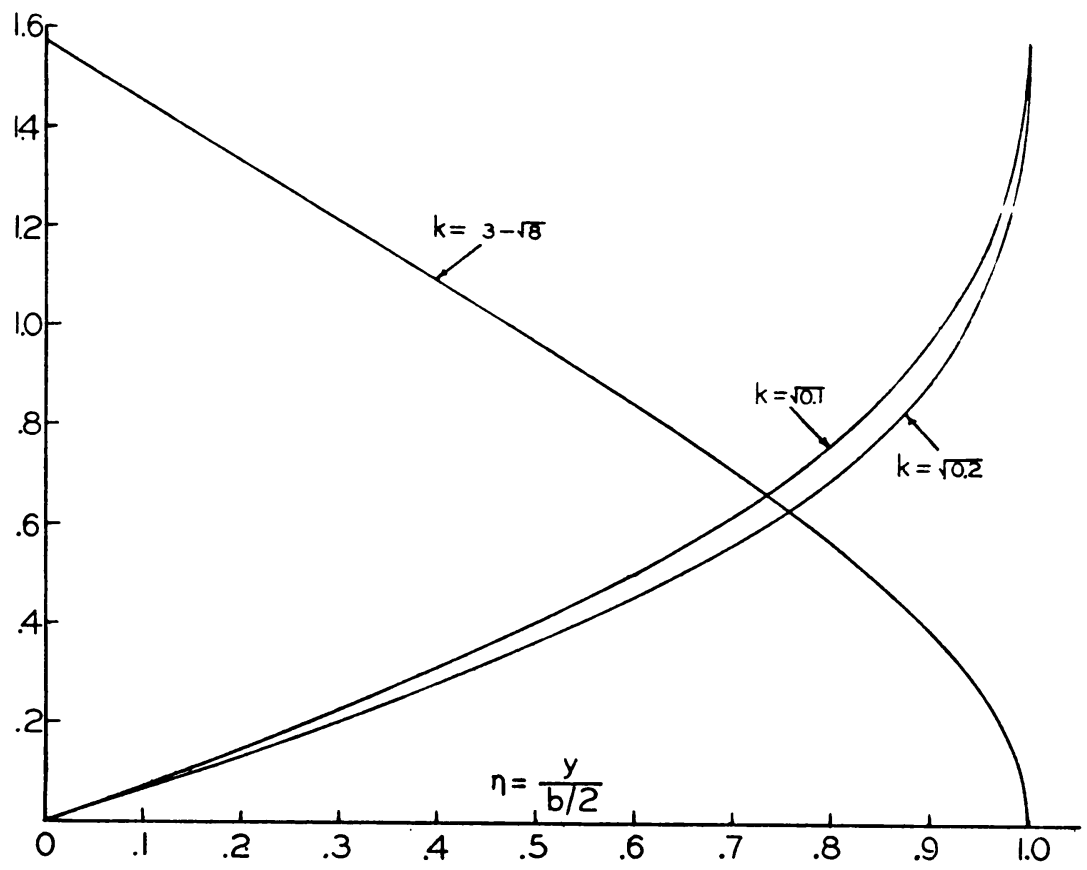

Fig. 5.

quick determination of the span coordinate associated with a certain coordinate value on the side of the rectangle of the boundary value problem. The two curves marked $k=\sqrt{ } .1$ and $k=\sqrt{ } .2$ represent plots of equation (34) and go with Tables 1 and 2.

TABLE I

Tapered Wing $k=\sqrt{.1}$

\begin{tabular}{|c|c|c|c|c|c|}
\hline \multirow[b]{2}{*}{$2 K X / \pi$} & \multirow[b]{2}{*}{$X$} & \multirow[b]{2}{*}{$\eta$} & \multicolumn{2}{|r|}{$c_{l} / m \alpha$} & \multirow[b]{2}{*}{$A / m=2$} \\
\hline & & & $A / m=1$ & $\mathcal{A} / m=1.5$ & \\
\hline 0 & 0 & 0 & 1.0191 & 1.1207 & 1.1807 \\
\hline .1 & .09742 & .13096 & 1.0070 & 1.1068 & 1.1655 \\
\hline .3 & .29225 & .37801 & .91652 & 1.0026 & 1.0527 \\
\hline .5 & .48709 & .58643 & .76447 & .82908 & .86553 \\
\hline .8 & .77934 & .80778 & .50527 & .53846 & .55574 \\
\hline 1.1 & 1.07159 & .93247 & .28109 & .29429 & .30021 \\
\hline
\end{tabular}

The third curve is a plot of equation $\left(34^{\prime}\right)$ and goes with Table 3. Figs. 6, 7, 8 show the dependence of lift coefficient upon aspect ratio and taper. They should be compared with Fig. 75 of Durand, vol. II (which was computed by the approximate method de- 


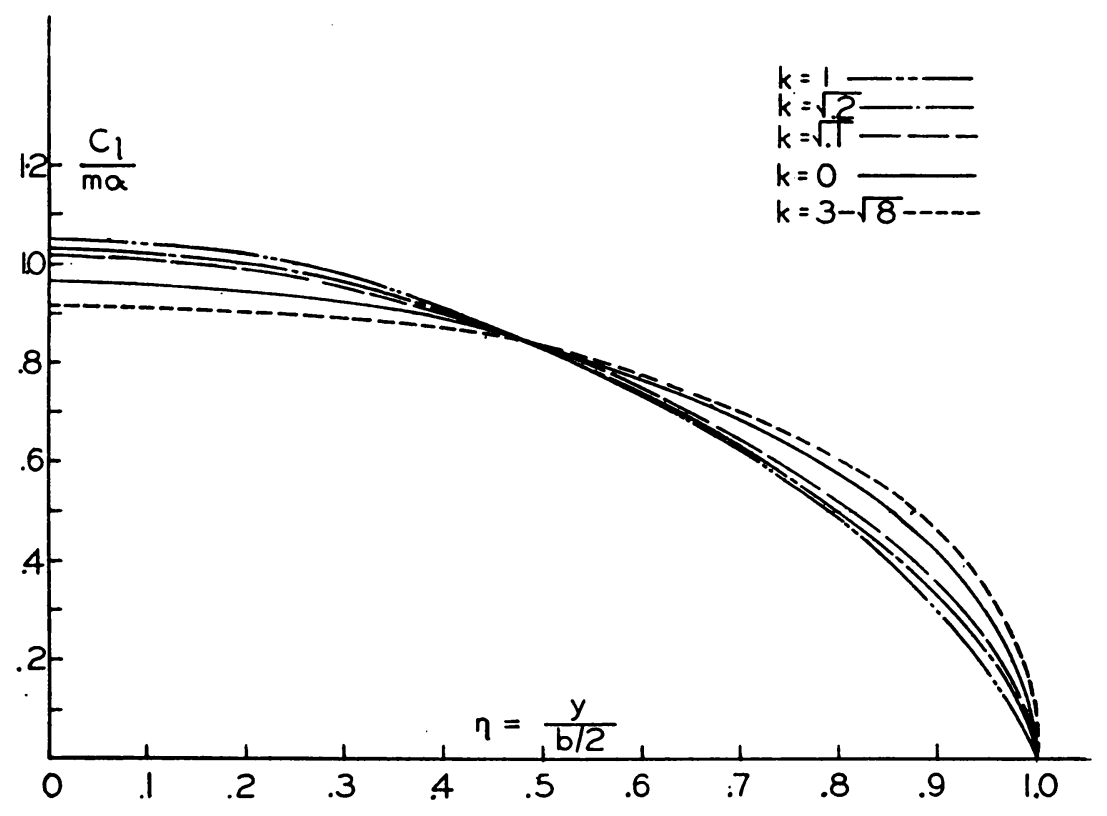

FIG. 6. $A / m=1.0$.

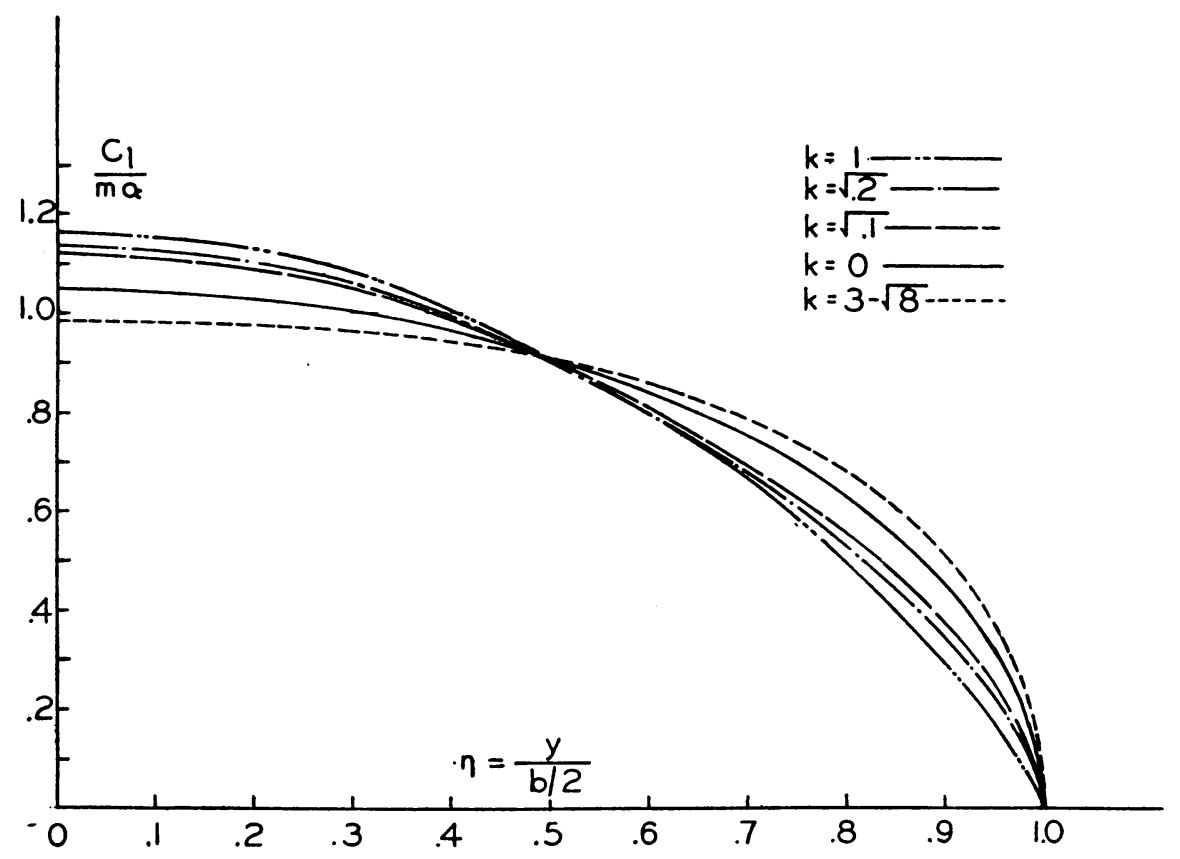

FIG. 7. $A / m=1.5$. 


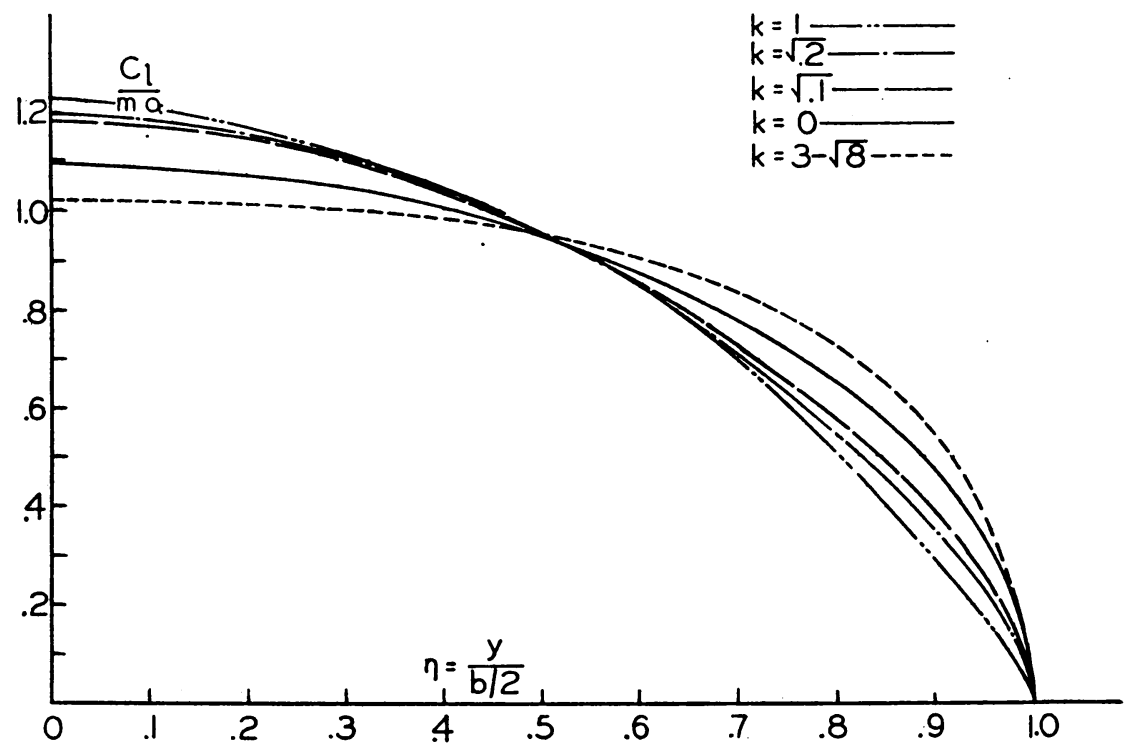

FIG. 8. $A / m=2.0$.

TABLE II

Tapered Wing $k=\sqrt{.2}$

\begin{tabular}{|c|c|c|c|c|c|}
\hline \multirow[b]{2}{*}{$2 K X / \pi$} & \multirow[b]{2}{*}{$X$} & \multirow[b]{2}{*}{$\eta$} & \multicolumn{3}{|c|}{$c_{l} / m \alpha$} \\
\hline & & & $A / m=1$ & $\mathcal{A} / m=1.5$ & $A / m=2$ \\
\hline 0 & 0 & 0 & 1.0306 & 1.1365 & 1.1987 \\
\hline .1 & .09465 & .14380 & 1.0150 & 1.1184 & 1.1792 \\
\hline .3 & .28394 & .41052 & .90134 & .98667 & 1.0370 \\
\hline .5 & .47324 & .62545 & .72028 & .77905 & .81219 \\
\hline .8 & .75718 & .83613 & .44105 & .46565 & .47700 \\
\hline 1.1 & 1.04113 & .94328 & .23139 & .23804 & .24100 \\
\hline
\end{tabular}

TABLE III

Blunt Wing $k=3-\sqrt{8}$

\begin{tabular}{c|c|c|c|c}
\hline & & \multicolumn{3}{|c}{$c_{l} / m \alpha$} \\
\cline { 2 - 4 }$\pi$ & $\eta$ & $A / m=1$ & $A / m=1.5$ & \multirow{A}{*}{$A / m=2$} \\
\hline$\pi / 2$ & 0 & .91288 & .98248 & 1.0205 \\
1.2 & .30920 & .88828 & .96045 & 1.0006 \\
1.0 & .47438 & .84591 & .91985 & .96191 \\
.7 & .70700 & .71481 & .78512 & .82654 \\
.5 & .83962 & .56705 & .62678 & .66262 \\
.35 & .91816 & .42178 & .46800 & .49601 \\
\hline
\end{tabular}


TABLE IV

Parabolic Wing $k=1.0$

\begin{tabular}{c|c|c|c|c}
\hline$X$ & \multicolumn{3}{|c}{} & \multicolumn{2}{c}{$c_{l} / m \alpha$} & \\
\hline 0 & $\eta$ & $A / m=1$ & $A / m=1.5$ & $A / m=2$ \\
\hline .1 & 0 & 1.050 & 1.164 & 1.230 \\
.2 & .19737 & 1.020 & 1.130 & 1.166 \\
.4 & .37995 & .9297 & 1.029 & 1.057 \\
.6 & .66404 & .66568 & .71789 & .74320 \\
.9 & .83365 & .42208 & .43141 & .44003 \\
& .94681 & .17295 & .17296 & .17297 \\
\hline
\end{tabular}

scribed in the introduction). The lift coefficient shows, of course, the expected span dependence, inasmuch as a more highly tapered wing gives rise also to a more highly tapered lift curve. Of special interest is the parabolic case, for which the lift coefficient goes to zero with infinite tangent although the chord function $c(y)$ has a finite tangent at the tips.

The author should like to thank Mr. H. Yoshihara for extensive assistance with the numerical calculations. A grant from the Faculty Research Fund of the University of Michigan is gratefully acknowledged. 